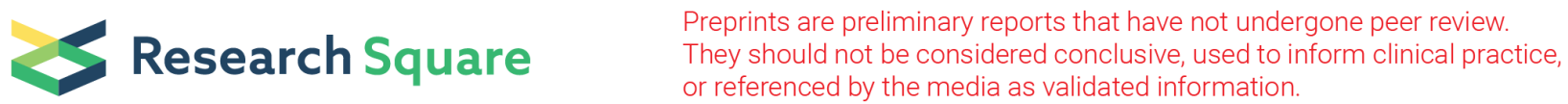 \\ Real-world analysis of age-dependent efficacy of COVID-19 vaccination
}

Giuseppe Lippi ( $\nabla$ giuseppe.lippi@univr.it )

Camilla Mattiuzzi

Brandon M. Henry

Short Report

Keywords: SARS-CoV-2, COVID-19, Vaccination, Efficacy

Posted Date: January 12th, 2022

DOI: https://doi.org/10.21203/rs.3.rs-1248612/v1

License: (c) (1) This work is licensed under a Creative Commons Attribution 4.0 International License.

Read Full License 


\title{
Real-world analysis of age-dependent efficacy of COVID-19 vaccination
}

\author{
Giuseppe Lippi ${ }^{1}$, Camilla Mattiuzzi ${ }^{2}$, Brandon M. Henry ${ }^{3,4}$
}

1. Section of Clinical Biochemistry and School of Medicine, University of Verona, Italy.

2. Service of Clinical Governance, Provincial Agency for Social and Sanitary Services, Trento, Italy.

3. Clinical Laboratory, Division of Nephrology and Hypertension, Cincinnati Children's Hospital Medical Center, Cincinnati, OH, USA.

4. Disease Intervention \& Prevention and Population Health Programs, Texas Biomedical Research Institute, San Antonio, Texas, USA.

Short title: Post-vaccine neutralization of SARS-CoV-2 Omicron

Word count: $1627+3$ Figures

Type of article: Short communication

\section{Corresponding author:}

Prof. Giuseppe Lippi

Section of Clinical Biochemistry

University Hospital of Verona

Piazzale L.A. Scuro, 10

37134 Verona - Italy

Tel. 0039-045-8122970

Fax. 0039-045-8124308

Email: giuseppe.lippi@univr.it 


\begin{abstract}
We analyze here data from the Italian coronavirus disease 2019 (COVID-19) vaccination campaign for providing real-world evidence on age-dependent efficacy of vaccination. Vaccine efficacy against infection, COVID-19 related hospitalization and intensive care unit (ICU) admission increased according to vaccination status, from people who received primary COVID-19 vaccination $>4$ months ago $(36 \%, 87 \%$ and 93\%, respectively), to those who received primary COVID-19 vaccination $<4$ months ago $(60 \%, 90 \%$ and $94 \%$, respectively), and finally to those with booster vaccine dose $(60 \%, 98 \%$ and $98 \%$, respectively). Vaccine efficacy (both primary and booster) against SARS-CoV-2 infection increased with ageing, though a similar trend could not be observed for hospitalization or ICU admission. Booster vaccine doses generated adjunctive protection against all endpoints compared to early primary vaccination in older people (i.e., aged >60 years). In conclusion, vaccine-elicited SARS-CoV-2 immunity declines over time, though vaccine boosters are effective for restoring immunity and conferring adjunctive protection.
\end{abstract}

Keywords: SARS-CoV-2; COVID-19; Vaccination; Efficacy 


\section{Introduction}

First appearing at the end of 2019 in the city of Wuhan (China) as a local outbreak of atypical pneumonia, the pathology that is now universally known as coronavirus disease 2019 (COVID-19) has spread all over the world, affecting over 300 million people and causing already 5.5 million deaths at the beginning of January 2022 according to statistics of the John Hopkins University Coronavirus Resource Center [1]. Despite the many physical preventive measures (i.e., social distancing, partial or total closures, use of face masks, hand hygiene and so forth) that have been put in place since the beginning of the severe acute respiratory syndrome coronavirus 2 (SARS-CoV-2) pandemics [2], the numbers of new COVID-19 cases and hospitalizations are still growing exponentially as a results of many concomitant factors, most significantly the appearance of new SARS-CoV-2 variants of concern (e.g., Omicron B.1.1.529), characterized by extremely higher infectivity and major capacity to evade the immune response [3].

Several lines of evidence now attest that widespread vaccination is the most effective strategy to limit the otherwise dramatic medical and social impact of SARSCoV-2 spread in the population. A number of meta-analyses have been published so far on vaccine efficacy for preventing symptomatic COVID-19 illness [4-7], which underpinned that vaccine efficacy may depend on several aspects such as the type of vaccine, demographical (age, sex, ethnicity, body mass index) and clinical (immunocompetence, co-morbidities) characteristics of recipients, number of doses and time elapsed since the last vaccine dose administration.

The impact of recipients' age on efficacy of COVID-19 vaccination remains an important issue, considering that the older and vulnerable population is that imposing the largest burden on healthcare due the higher risk of developing severe/critical forms of COVID-19 [8]. A recent meta-analysis published by Wang and co-authors concluded 
that the SARS-CoV-2 geometric mean titer measured by live virus neutralization test in vaccinated older adults (i.e., aged 55 years or older) was significantly higher than that found in younger vaccine recipients, thus raising important doubts as to whether the immunogenicity achieved after completing a primary vaccination may need additional "boosters" for obtaining a sufficient level of protection against the unfavourable consequences of this new and life-threatening infection in older people [5]. This straightforward concept, attributable to the so-called immunosenescence, has been more recently reaffirmed by Collier et al. [9], who also evidenced that vaccine-elicited humoral and cellular immunity appears significantly lower in recipients aged 80 years or older.

To provide real-world evidence on the age-dependent efficacy of COVID-19 vaccination, we provide here an updated analysis of the Italian nationwide vaccination campaign.

\section{Materials and Methods}

We accessed the official data emerging from the Italian nationwide COVID-19 vaccination campaign and published on a weekly basis by the Italian National Institute of Health (Istituto Superiore di Sanità, ISS; Last available update, January 7, 2022). The official bulletin of the ISS contains update statistics on COVID-19 vaccinations (primary and/or booster doses), as well as on total number of new diagnoses of SARSCoV-2 infection, COVID-19 related hospitalizations and intensive care unit (ICU) admissions in the country, presented as a whole and clustered by age ranges (i.e., 12-39, $40-59,60-80$ and $>80$ years) $[10]$.

Vaccine efficacy was expressed as odds ratio (OR) with $95 \%$ confidence interval (95\%CI) of SARS-CoV-2 infection, COVID-19 related hospitalization and COVID-19 related intensive care unit admission across different age cohorts, and was estimated 
using MetaXL, software Version 5.3 (EpiGear International Pty Ltd., Sunrise Beach, Australia). This study was conducted in accordance with the Declaration of Helsinki, under terms of relevant local legislation. The research was based on freely and publicly available ISS data, such that Ethical Committee approval was not required.

\section{Results}

At the time of our updated analysis (i.e., January 7, 2022) a total number of 37.668 million of Italian citizens had already completed a primary vaccination cycle (26.019 million over 4 months ago and 11.648 million within 4 months since the last primary vaccine dose), 8.102 million had received a vaccine booster dose, whilst 6.660 million citizens were still unvaccinated. Primary vaccination coverage was as follows: Pfizer/BionTech (mRNA-based) 67.1\%, Moderna (mRNA-based) 21.0\%, AstraZeneca (Adenovirus-based) $10.5 \%$ and Janssen (Adenovirus-based) 1.3\%, respectively.

The results of our analysis are shown in Figures 1 to 3 . Vaccine efficacy against development of SARS-CoV-2 infection gradually increased from $36 \%$ in people who had received primary COVID-19 vaccination over 4 months ago (OR 0.64; 95\%CI, $0.42-0.99)$, to $60 \%(\mathrm{OR}, 0.40 ; 95 \% \mathrm{CI}, 0.29-0.55)$ in those who had received primary COVID-19 vaccination within 4 months as well as in those who had received a booster vaccine dose (OR, 0.40; 95\% 0.22-0.72) (Figure 1). Notably, the efficacy of vaccination (both primary and booster dose administration) against development of SARS-CoV-2 infection increased in parallel with ageing, being the lowest in the younger cohort (1239 years; $15-55 \%)$ and the highest in those aged 80 years or older $(72-86 \%)$.

As shown in Figure 2, vaccine efficacy against COVID-19 related hospitalization also gradually increased from $87 \%$ in people who had received primary COVID-19 vaccination over 4 months ago (OR 0.13; 95\%CI, 0.08-0.23), to 90\% (OR, 0.10; 95\%CI, 0.06-0.16) in those who had received primary COVID-19 vaccination 
within 4 months, up to $98 \%$ in those who had received a booster vaccine dose (OR, 0.02; 95\% 0.01-0.03). Unlike the effectiveness against SARS-CoV-2 infection, vaccine efficacy against COVID-19 related hospitalization did not display a clear age-related trend, appearing the lowest (i.e., $79 \%$ and $87 \%$ ) in people aged $60-80$ or $>80$ years who had received primary COVID-19 vaccination by over 4 months, but then becoming the highest in these same age groups after receiving the booster dose (i.e., $98 \%$ in both cases).

Vaccine efficacy against COVID-19 related ICU admission is shown in Figure 3. As for the previous endpoints, vaccine efficacy progressively increased from $93 \%$ in people who had received primary COVID-19 vaccination over 4 months ago (OR 0.0.7; $95 \% \mathrm{CI}, 0.03-0.16)$, to $94 \%(\mathrm{OR}, 0.06 ; 95 \% \mathrm{CI}, 0.03-0.13)$ in those who had received primary COVID-19 vaccination within 4 months, up to $98 \%$ in those who had received a booster vaccine dose $(\mathrm{OR}, 0.02 ; 95 \%$ 0.01-0.02). As for hospitalizations, vaccine efficacy did not display a clear age-related trend for odds of ICU admission, appearing the lowest (i.e., $93 \%$ and $87 \%$ ) in people aged $60-80$ or $>80$ years who had received primary COVID-19 vaccination over 4 months ago, but then becoming the highest in this same age group after receiving the booster dose (i.e., $99 \%$ in both cases).

\section{Discussion}

With the refurbished and unremitting spread of SARS-CoV-2 infections, hospitalizations and ICU admissions recorded over recent times all around the world [1], the laboratory and medical burden for diagnosing and managing new COVID-19 cases is becoming more challenging than ever [11]. Although the recently emergent Omicron variant seems to be associated with lower severity of COVID-19 illness, its extremely magnified infectivity (over 3-fold that of the former Delta B.1.617.2 strain) is enormously amplifying the number of SARS-CoV-2 infections and, consequently, the 
risk that more vulnerable individuals may need hospital care [12]. It is hence obvious that whatever strategy aimed at preventing or limiting the circulation of the virus and its unfavourable impact on vulnerable individuals, shall be regarded as a primary healthcare issue. To this end, verification of efficacy of COVID-19 vaccination in different age classes of the general population is essential in the face of a dramatic shortages of material and human resources for supporting (or even maintaining) ongoing worldwide vaccination and booster campaigns [13].

In our analysis, in keeping with previous reports, we observed vaccine efficacy was found to clearly wane over time, with protection against all of the clinical endpoints that we analysed in this study declining 4 months after completion of the primary vaccination cycle. This decline appeared especially accentuated in older individuals (i.e., aged 60 years or older), wherein vaccine efficacy against the risk of COVID-19 related hospitalization and ICU admission decreased from $87 \%$ to $79-87 \%$ and from 91 $92 \%$ to $87-93 \%$, respectively, after 4 months from the last primary vaccine dose. However, vaccine efficacy against both endpoints was considerably enhanced to $\geq 98 \%$ in older persons who received the booster vaccine dose. This aspect not only supports the practice of restoring immune protection by administration of periodic vaccine boosters (e.g., at least 4 months after completing the primary vaccination), but provides further evidence that adjunctive vaccine doses may generate larger protection against hospitalization and ICU admission compared to the standard primary vaccination.

Besides the favourable impact on reducing hospital pressure driven by COVID19 hospitalizations and ICU admissions, the administration of vaccine booster doses appeared also to be effective for reducing viral circulation compared to vaccine effectiveness recorded more than 4 months after primary vaccination (i.e., $60 \%$ vs. $36 \%$ ), though no clear adjunctive effect on infection could be noted comparing primary vaccination within 4 months vs. the booster in the total population (i.e., $40 \%$ vs. $40 \%$ ). 
Such results on infection may be biased, as recently vaccinated or boosted individuals may be less likely to by symptomatic and seek testing. Nonetheless, the booster vaccine dose was effective to further lower the odds of SARS-CoV-2 infection in the older population (i.e., from $70 \%$ to $75 \%$ in those aged $60-80$ years and from $81 \%$ to $86 \%$ in those aged 80 years or older, respectively), thus bringing obvious advantages to this more vulnerable part of the population, in whom early diagnosis is mandatory to enable interventions to prevent disease progression and in whom even mild/moderate viral infections may have substantial clinical consequences.

\section{Conclusion}

In conclusion, the results of our analysis confirm that vaccine-elicited SARSCoV-2 immunity declines over time [14], though we also support the concept that vaccine boosters may not only be effective for restoring immunity [15], but also for conferring adjunctive protection against the risk of infection and any type of COVID-19 related hospitalization, especially in the older population.

\section{Authors' contribution}

Study design: GL, CM, BMH; Data Collection: GL, CM; Data analysis: GL, BMH; Writing: GL, BMH.

\section{Authorship criteria}

All authors attest they meet the ICMJE criteria for authorship.

\section{Funding}

None declared. 


\section{Declaration of Competing Interest}

The authors declare that they have no known competing financial interests or personal relationships that could have appeared to influence the work reported in this paper.

\section{Acknowledgments}

None.

\section{References}

1. John Hopkins University. Coronavirus Resource Center Available at: https://coronavirus.jhu.edu/map.html. Last accessed, January 10, 2022.

2. Iezadi S, Gholipour K, Azami-Aghdash S, Ghiasi A, Rezapour A, Pourasghari H, et al. Effectiveness of non-pharmaceutical public health interventions against COVID19: A systematic review and meta-analysis. PLoS One 2021;16:e0260371.

3. Lippi G, Mattiuzzi C, Henry BM. Neutralizing potency of COVID-19 vaccines against the SARS-CoV-2 Omicron (B.1.1.529) variant. J Med Virol. 2022 Jan 5. doi: 10.1002/jmv.27575.

4. Rotshild V, Hirsh-Raccah B, Miskin I, Muszkat M, Matok I. Comparing the clinical efficacy of COVID-19 vaccines: a systematic review and network meta-analysis. Sci Rep 2021;11:22777.

5. Wang J, Tong Y, Li D, Li J, Li Y. The Impact of Age Difference on the Efficacy and Safety of COVID-19 Vaccines: A Systematic Review and Meta-Analysis. Front Immunol 2021;12:758294.

6. Sharif N, Alzahrani KJ, Ahmed SN, Dey SK. Efficacy, Immunogenicity and Safety of COVID-19 Vaccines: A Systematic Review and Meta-Analysis. Front Immunol 2021;12:714170. 
7. Zheng C, Shao W, Chen X, Zhang B, Wang G, Zhang W. Real-world effectiveness of COVID-19 vaccines: a literature review and meta-analysis. Int J Infect Dis 2022;114:252-60.

8. Singhal S, Kumar P, Singh S, Saha S, Dey AB. Clinical features and outcomes of COVID-19 in older adults: a systematic review and meta-analysis. BMC Geriatr $2021 ; 21: 321$.

9. Collier DA, Ferreira IATM, Kotagiri P, Datir RP, Lim EY, Touizer E, et al. Agerelated immune response heterogeneity to SARS-CoV-2 vaccine BNT162b2. Nature 2021;596:417-22.

10. Istituto Superiore di Sanità. Epidemia COVID-19. Last update: January 7, 2022. Last accessed, January 10, 2022.

11. Tanne JH. Covid-19: US hospitals are overwhelmed as cases rise at "alarming rate". BMJ 2021;374:n2092.

12. Ito K, Piantham C, Nishiura H. Relative Instantaneous Reproduction Number of Omicron SARS-CoV-2 variant with respect to the Delta variant in Denmark. J Med Virol. 2021 Dec 30. doi: 10.1002/jmv.27560. Epub ahead of print.

13. Hassan F, London L, Gonsalves G. Unequal global vaccine coverage is at the heart of the current covid-19 crisis. BMJ 2021;375:n3074.

14. Khoury J, Najjar-Debbiny R, Hanna A, Jabbour A, Abu Ahmad Y, Saffuri A, et al. COVID-19 vaccine - Long term immune decline and breakthrough infections. Vaccine 2021;39:6984-9.

15. Bar-On YM, Goldberg Y, Mandel M, Bodenheimer O, Freedman L, Alroy-Preis S, et al. Protection against Covid-19 by BNT162b2 Booster across Age Groups. N Engl J Med 2021;385:2421-30. 
Figure 1. Efficacy of coronavirus disease 2019 (COVID-19) vaccination against the risk of developing SARS-CoV-2 (severe acute respiratory syndrome coronavirus 2) infection.
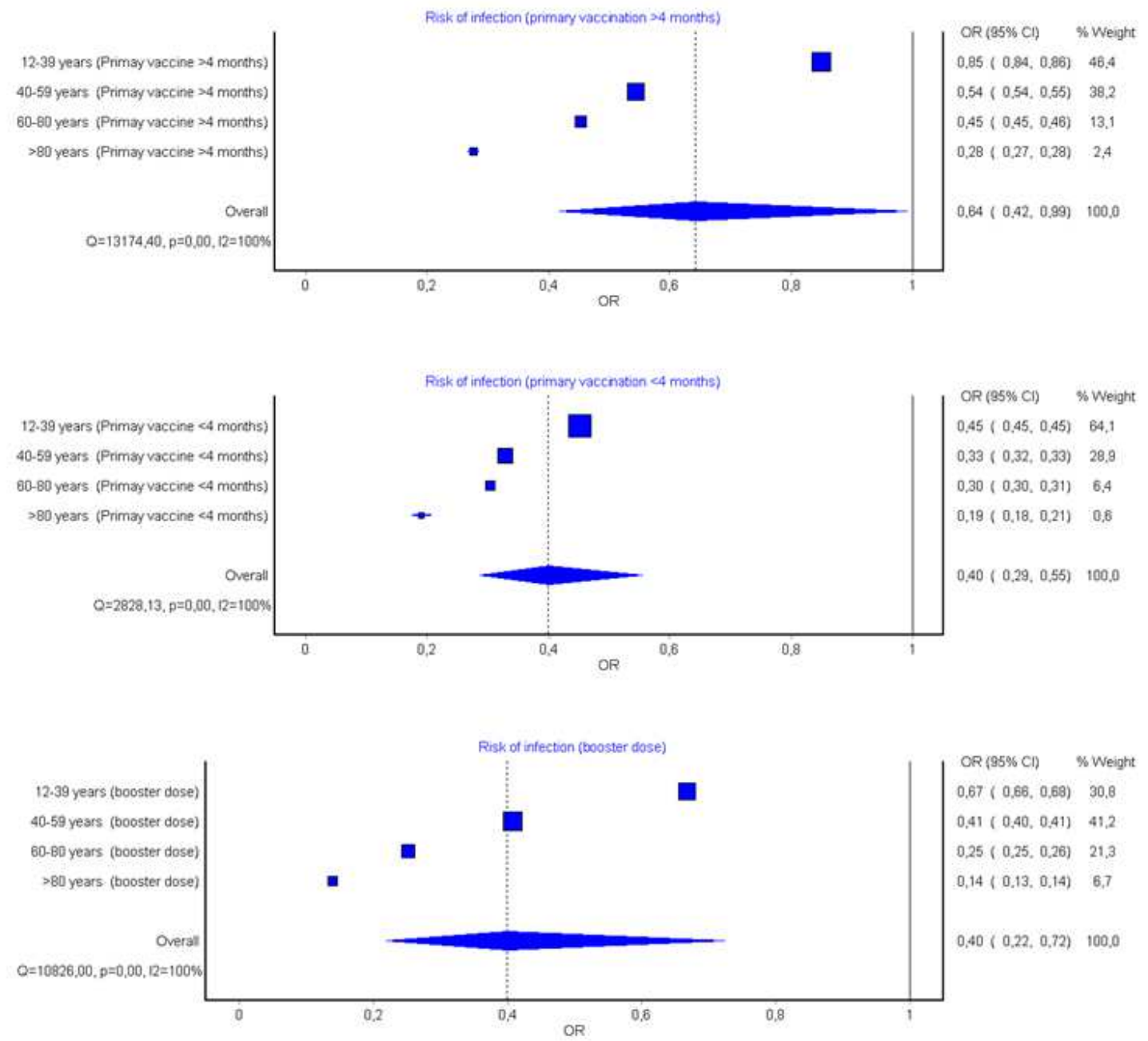

OR, odds ratio; 95\%CI, 95\% confidence interval 
Figure 2. Efficacy of coronavirus disease 2019 (COVID-19) vaccination against the risk of COVID-19 related hospitalization.
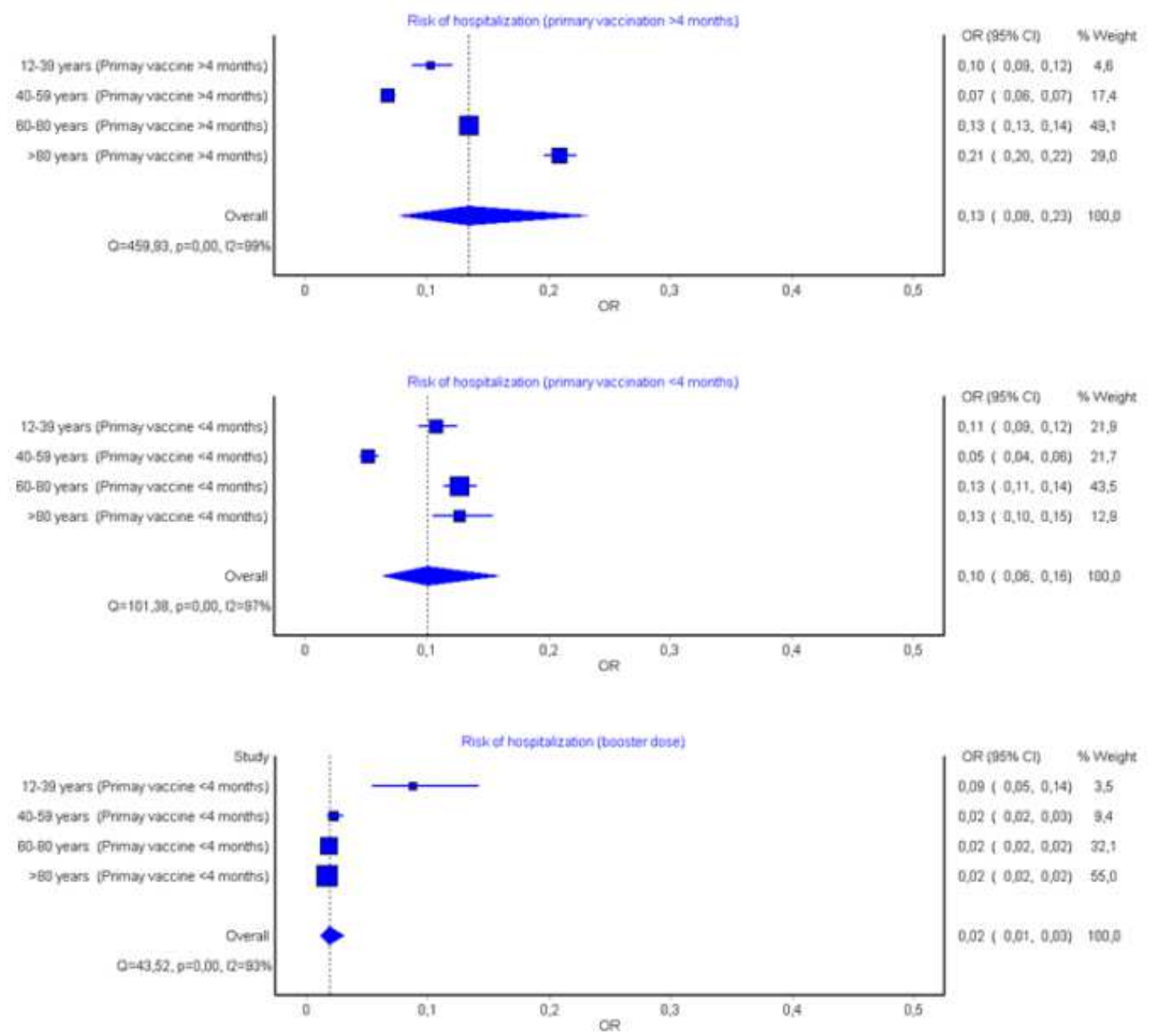

OR, odds ratio; 95\%CI, 95\% confidence interval 
Figure 3. Efficacy of coronavirus disease 2019 (COVID-19) vaccination against the risk of COVID-19 related intensive care unit (ICU) admission.
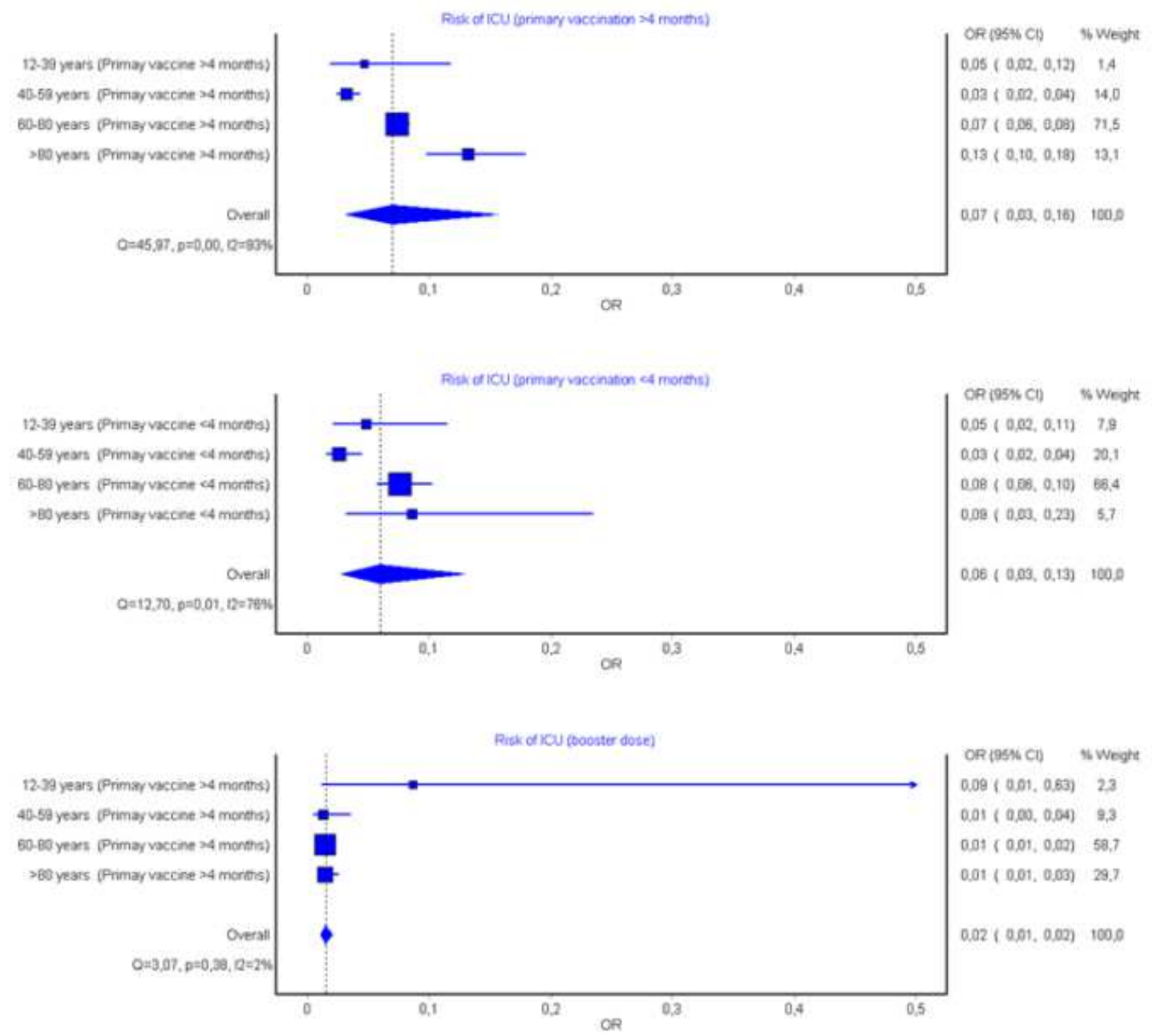

OR, odds ratio; 95\%CI, 95\% confidence interval 